\title{
Compositions of Anticancer Drug with Micellar Nanocarriers and Their \\ Cytotoxicity
}

Larisa R. Kunitskaya $^{\mathrm{a}^{*} \text {, Tatyana B. Zheltonozhskaya }}{ }^{\mathrm{a}}$, Rostyslav S. Stoika ${ }^{\mathrm{b}}$, Dmytro O. Klymchuk ${ }^{\mathrm{c}}$

${ }^{a}$ Department of Chemistry, Taras Shevchenko National University of Kyiv, Volodymyrska Street, 64/13, Kyiv 01601, Ukraine

${ }^{\mathrm{b}}$ Institute of Cell Biology NAS of Ukraine, 14/16 Dragomanov St., Lviv 79005, Ukraine

${ }^{\mathrm{c}}$ Institute of Botany NAS of Ukraine, 2 Tereschenkivska Str., Kyiv 01601, Ukraine

contactinge-mail: larisa_kunitskaya@ukr.net

Keywords: micelles, morphology, block copolymers, doxorubicin, cytotoxicity

Asymmetric diblock (DBC) and triblock (TBC) copolymers contained biocompatible chemically complementary polyacrylamide and poly(ethylene oxide) (PAAm- $b$-PEO- $b$-PAAm) or its monomethyl ether (MEPEO- $b$-PAAm), and also partially hydrolyzed triblock copolymer derivative $\left(\mathrm{TBC}_{\mathrm{hydr}}\right)$ were used to create micelles of a special type. The micelles obtained are characterized by small CMCs and large values of the Gibbs micellization energy, thus indicating a high stability of $\mathrm{DBC}, \mathrm{TBC}$ and $\mathrm{TBC}_{\text {hydr }}$ micelles in aqueous solutions and the capabilities of their use to encapsulate and deliver poorly soluble and/or toxic drugs in living organism. Morphological features and size of DBC and TBC micelles were determined by TEM. The electron images demonstrated spherical micelles of a polymolecular type, monomolecular type and separate micelle aggregates. $\mathrm{TBC}$ and $\mathrm{TBC}_{\text {hydr }}$ micelles were used to examine in vitro anticancer activity of their compositions with doxorubicin (Dox). The created micelle systems showed the enhanced cytotoxicity as compared to individual Dox against murine leukemia cells of L1210 line, murine transformed fibroblasts of L929 line and human T-leukemia cells of Jurkat line and allow to achieve a high efficacy at low Dox concentrations $\left(0,1 \div 3 \mu \mathrm{g} \cdot \mathrm{cm}^{-3}\right)$ that opens the great prospects for essential decrease in drug dose at chemotherapy.

\section{Introduction}

Polymeric micellar nanocontainers for encapsulating and delivering drugs and biopolymers in living organisms allow: i) creating water-soluble forms of poorly soluble drugs, ii) ensuring a long-term circulation of drugs in a bloodstream, iii) preventing the rapid degradation of drugs in the enzymatic and metabolic processes, iv) reducing essentially therapeutic doses of drugs, v) protecting healthy organs and tissues of the body from the destructive action of toxic drugs, especially at cancer chemotherapy, and vi) avoiding the immune system response to the introduction of 
foreign proteins and nucleic acids [1-8]. In the presence of special ligands (vectors), such micellar nanocontainers are capable of targeted drug delivering and providing their controlled release in certain organs, tissues or cells [9-13]. Thus, the polymeric micelles can be considered as one of the most convenient and promising delivery vehicles.

In a clinical practice, doxorubicin (Dox) is widely applied for cancer treatment due to powerful action on a wide range of tumour cells. However, a high toxicity of the drug limits its use because of serious adverse complications, especially in the cardiovascular system [14-15]. That is why in recent years, much attention is devoted to the development of suitable micelle carriers for Dox delivery, which can improve its therapeutic efficacy and minimize side effects. Initially, to create the polymeric micelle carriers the classical amphiphilic block copolymers capable of self-assembly in aqueous medium, were used. They were generally based on the blocks of poly(ethylene oxide) and poly(propylene oxide), polyaminoacids, polycaprolactone, polylactides, etc. [11,16-17]. During past decade a number of polymeric micelles with the cross-linked "core" were presented as potential nanocarries for Dox. Among them, there are the cross-linked micelles based on poly(ethylene oxide)- $b$ poly(methacrylic acid), poly(ethylene oxide)- $b$ poly(e-caprolactone) and poly(hydroxyethyl acrylate)-b-poly(n-butyl acrylate) block copolymers [18-20].

In present study, the polymeric micelles of a special type were examined as potential carriers for Dox. They were formed by the asymmetric diblock (DBC) and triblock (TBC) copolymers contained biocompatible chemically complementary polyacrylamide and poly(ethylene oxide) (PAAm- $b$-PEO-b-PAAm) or its monomethyl ether (MEPEO-b-PAAm), and also the partially hydrolyzed triblock copolymer derivative $\left(\mathrm{TBC}_{\text {hydr }}\right)$. It should be noted that (ME)PEO and PAAm chains are known as non-toxic and biocompatible polymeric components; the first of them can even be biodegraded in living organisms. Therefore, the block copolymers consisting of such blocks could successfully be used in the biomedicine. Peculiarities of the DBC, TBC and $\mathrm{TBC}_{\text {hydr }}$ self-assembly in aqueous solutions, a size and morphology of the obtained micelles, as well as in vitro cytotoxicity of some their compositions with Dox have been highlighted.

\section{Experimental part}

\section{Materials}

Monomethyl ether of poly(ethelene glycol) (MEPEG) with $\mathrm{M}_{\mathrm{n}}=5 \mathrm{kDa}$ from "Fluka" (Germany) as well as poly(ethylene glycol) (PEG) with $\mathrm{M}_{\mathrm{n}}=6 \mathrm{kDa}$ and ammonium cerium (IV) nitrate from "Aldrich" (USA) were used as received. Monomer acrylamide (AAm) produced by "Merck" (Germany) was twice recrystallized from chlorophorm before the block 
copolymer syntheses. Doxorubicin was purchased from "Veropharm" Company (Russia) and used in the hydrochloride form.

\section{General}

\section{Procedure}

\section{Copolymerization Process}

DBC and TBC samples were prepared by the radical block copolymerization of polyacrylamide (PAAm) to MEPEG or PEG initiated by $\mathrm{Ce}^{\mathrm{IV}}$ ions at $20^{\circ} \mathrm{C}$ according to the technique described in our previous papers $[21,22]$. The block copolymerization was carried out at the molar ratios $\left[\mathrm{Ce}^{\mathrm{IV}}\right] /[-$ $\mathrm{OH}]=1 \div 1,04$ and $\left[\mathrm{Ce}^{\mathrm{IV}}\right] /[\mathrm{AAm}]=1 \cdot 10^{-3}$ in the deionized water and inert atmosphere during 24 $h$. The synthesized samples of TBC were reprecipitated by acetone, then dissolved in the deionized water and freeze dried.

Partial Hydrolysis of Polyacrylamide

\section{Blocks}

The partially hydrolysed triblock copolymer $\left(\mathrm{TBC}_{\mathrm{hydr}}\right)$ was obtained from TBC sample by the alkaline hydrolysis reaction of acrylamide units. TBC solution in the dionized water with concentration $\mathrm{C}_{\mathrm{TBC}}=10$ $\mathrm{kg} \cdot \mathrm{m}^{-3}$ was stirred in the presence of $\mathrm{NaOH}$ $\left(\mathrm{C}_{\mathrm{NaOH}}=5 \mathrm{~mol} \cdot \mathrm{dm}^{-3}\right)$ at $\mathrm{T}=50{ }^{\circ} \mathrm{C}$ for $10 \mathrm{~min}$. The $\mathrm{Na}^{+}$-form of the modified triblock copolymer was transformed into $\mathrm{H}$-form by treating with $0.5 \mathrm{~N} \mathrm{HCl}$ to $\mathrm{pH} 2$. The gel-like $\mathrm{TBC}_{\text {hydr }}$ was re-precipitated by acetone, dissolved in water and freeze-dried. The hydrolysis degree of acrylamide units in the modified copolymer (10.7 mol \%) was determined using

potentiometric titration of the copolymer solution in pure water by $\mathrm{NaOH}$ and calculation of the hydroxyl ions absorption curve.

Characterization of Chemical Structure and Molecular Parameters

Chemical structure of the copolymers and the number-average molecular weights of PAAm blocks were determined from ${ }^{1} \mathrm{H}$ NMR spectra, which were recorded in D2O at $\mathrm{C}=1 \mathrm{~kg} \cdot \mathrm{m}^{-3}$ and a room temperature using a Varian Mercury-400 spectrometer operating at $400 \mathrm{MHz}$. The chemical shifts

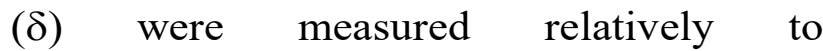
tetramethylsilane as a standard.

Static Light Scattering

\section{Measurements}

The critical micellization concentration (CMC) for the obtained block copolymers in aqueous medium was determined by static light scattering (SLS) using a modernized light scattering instrument FRS-3 (Russia) contained WP7113VGC/A light-emitting diode ( $\lambda=520$ $\mathrm{nm})$ from "Kingbright", ADC-CPUTM controller from "Insoftus", (Ukraine) and the computer program WINRECODER. In order to define CMCs, the scattering intensities of the vertically polarized light were measured in a wide region of the copolymer concentrations at the $\theta=90^{\circ}$ scattering angle and $\mathrm{T}=20^{\circ} \mathrm{C}$. 


\section{Transmission electron microscopy} (TEM)

TEM images of $\mathrm{DBC}, \mathrm{TBC}$ and TBC $C_{\text {hydr }}$ micelles formed in aqueous solutions were recorded with a JEM-I23O instrument ("JEOL', Japan) operating at an accelerating voltage of $90 \mathrm{kV}$. Small drops $\left(\sim 1 \cdot 10^{-4} \mathrm{~cm}^{3}\right)$ of the dilute solutions of TBC micelles $\mathrm{C}_{\mathrm{TBC}}=0.2 \mathrm{~kg} \cdot \mathrm{m}^{-3}$ ) prepared in the deionized water, were deposited in copper grids coated with Formvar film and carbon, and then were dried at a room temperature in air for $0.5-1 \mathrm{~min}$ and in vacuum desiccator at $0.2-0.3 \mathrm{~Pa}$ for $24 \mathrm{~h}$.

\section{Fourier Transform Infrared (FTIR)} Spectroscopy

FTIR spectra of TBC, DBC and PAAm were recorded using a Nexus-470 Nicolet (USA) spectrometer with a resolution $4 \mathrm{~cm}^{-1}$. All the spectra were recalculated in the dependences of the optical density (D) versus the wavenumber (v) using the relation: $\mathrm{D}=\log \mathrm{T}_{0} / \mathrm{T}$, where $\mathrm{T}_{0}$ and $\mathrm{T}$ are the maximum and current values of the transmittance in a certain spectrum.

\section{Ultraviolet-Visible (UV-Vis) Spectroscopy}

UV spectra of Dox, DBC and Dox/DBC aqueous solutions were recorded on a UV-Vis spectrometer Perkin Elmer Lambda 20 (Sweden) by scanning in the range 200-1000 nm. Dilute aqueous solutions were examined in quartz cuvettes, using pure solvent as a reference.

\section{Cytotoxicity studies in vitro}

Cytotoxicity in vitro of the

Dox/micelles compositions was investigated using murine leukemia cells of L1210 line, murine transformed fibroblasts of L929 line and human T-leukemia cells of Jurkat line. The L1210 and L929 cells were incubated in DMEM medium (Dulbecco's modified Eagle's medium) while Jurkat cells were incubated in RPMI medium (Roswell Park Memorial Institute medium) in the presence of $10 \%$ bovine serum. Incubation was performed in the 96-well plates ("Falcon", USA) placed in the $\mathrm{CO}_{2}$-incubator ("JENCONS NUAIRE", UK), in the atmosphere composed of air $(95 \mathrm{v} \%)$ and $\mathrm{CO}_{2}(5 \mathrm{v} \%)$. We compared the action of free Dox, Dox/micelles compositions, and free micelles. The half-lethal dose $\left(\mathrm{LD}_{50}\right)$ of Dox for the above-mentioned cell lines was 1 $\mu \mathrm{g} \cdot \mathrm{cm}^{-3}$. The effectiveness of the investigated preparations was determined as the number of living cells relatively to control (the number of alive cells in culture medium without the drug). The calculation of cell number was carried out in hemocytometer chamber in 24 and 48 hours after incubation beginning. Trypan blue was used to count the dead cells. In all cases we found the average numbers of living cells from three parallel experiments.

\section{Results and discussion}

Previously, it was found that the process 


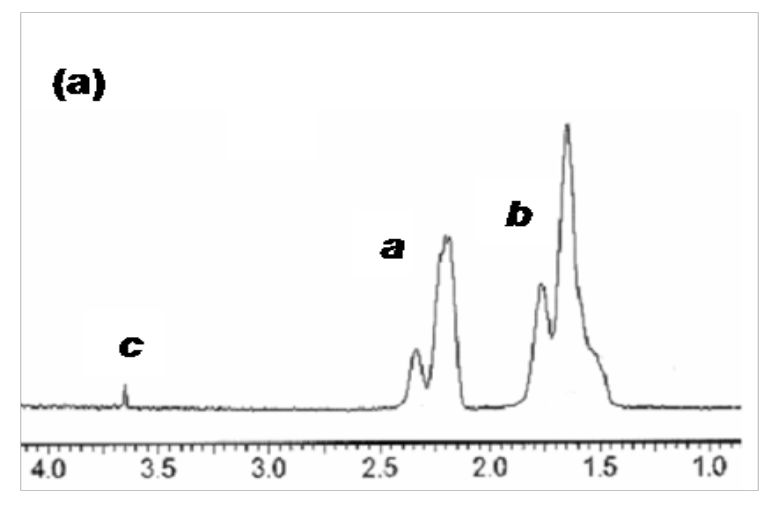

$\delta$, ppm

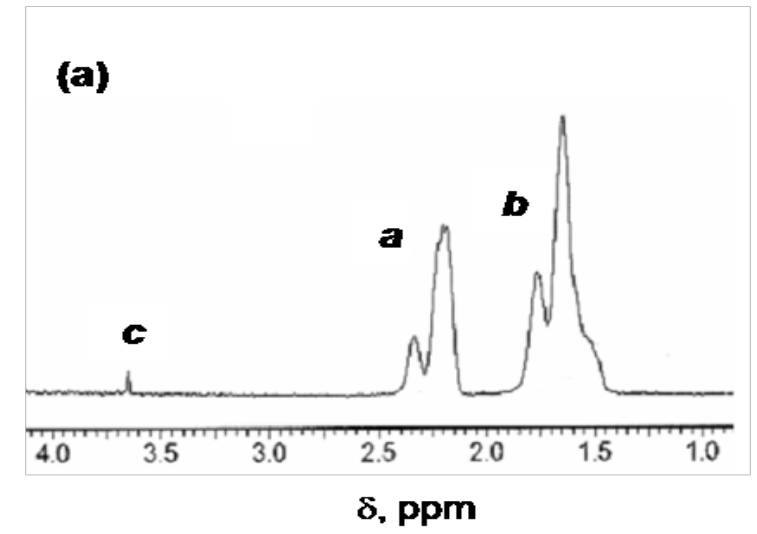

Figure 1. ${ }^{1} \mathrm{H}$ NMR spectra of (a) DBC and (b) TBC samples in $\mathrm{D}_{2} \mathrm{O} \cdot \mathrm{C}_{\mathrm{DBC} / \mathrm{TBC}}=10 \mathrm{~kg} \cdot \mathrm{m}^{-3}, \mathrm{~T}=20^{\circ} \mathrm{C}$

of block copolymerization of PAAm with (ME)PEG were characterized by the dynamic template effects which consisted in the changing the copolymerization rate and monomer conversion, as compared to PAAm homopolymerization performed in the same experimental conditions, and also by the structural template effect, namely, the growth of PAAm block length with increasing the length of the template (ME)PEO blocks [21, 22]. This was conditioned by the formation of cooperative hydrogen bonds between (ME)PEO chains and growing PAAm "daughter's" chains [23]. The value and size (positive or negative) of the dynamic template effects depended in a complicated manner upon the length of (ME)PEO blocks and concentration of the initiator and monomer. The synthesized DBCs and TBCs were soluble in water but their solutions demonstrated a slight opalescence depending on concentration due to the interaction of chemically complementary blocks, thus initiating the process of micelle formation.

${ }^{1} \mathrm{H}$ NMR spectra recorded in $\mathrm{D}_{2} \mathrm{O}$ for two DBC and TBC samples, synthesized in this work, are shown in Figure 1. Proton signals of $>\mathrm{CH}-$ (a) and $-\mathrm{CH}_{2}-$ groups (b) in PAAm blocks were found with the chemical shifts $\delta=2.1-2.4$ and 1.4-1.9 ppm, while the proton signal of $-\mathrm{CH}_{2}$ - groups (c) in (ME)PEO blocks had the values $\delta=3.70$ ppm. In the spectrum of DBC (Figure $1 \mathbf{a}$ ), a weak proton signal of the terminal $-\mathrm{OCH}_{3}$ groups $(\delta=3.38 \mathrm{ppm})$ was observed.

The number-average molecular weights of PAAm blocks in DBC and TBC samples were calculated using corresponding integrated intensities of the proton signals and the (2) equations:

$$
\begin{gathered}
M_{n P A A m(D B C)}=\frac{2 \cdot M_{o P A A m} \cdot M_{n M E P E O} \cdot A_{b}}{M_{o M E P E O} \cdot A_{c}} \\
M_{n P A A m(T B C)}=\frac{M_{o P A A m} \cdot M_{n P E O} \cdot A_{b}}{A_{c}}
\end{gathered}
$$


Table 1 Molecular parameters of the block copolymers according to ${ }^{1} \mathrm{H}$ NMR spectroscopy

\begin{tabular}{|c|c|c|c|c|c|}
\hline Copolymer & $\mathrm{M}_{\mathrm{n}(\mathrm{ME}) \text { PEO }}, \mathrm{KDa}$ & $\mathrm{M}_{\mathrm{nPAAm}}, \mathrm{KDa}$ & $\mathrm{M}_{\mathrm{nDBC} / \mathrm{TBC},} \mathrm{KDa}^{1)}$ & $\mathrm{w}_{(\mathrm{ME}) \text { PEO }}, \%^{2)}$ & $\mathrm{n}^{3)}$ \\
\hline $\mathrm{DBC}$ & 5 & 222 & 227 & 2.2 & 27,6 \\
\hline $\mathrm{TBC}$ & 6 & 116 & 238 & 2.5 & 12,1 \\
\hline
\end{tabular}

1) The molecular weight of TBC was calculated as: $\mathrm{M}_{\mathrm{nTBC}}=\mathrm{M}_{\mathrm{nPEO}}+2 \cdot \mathrm{M}_{\mathrm{nPAAm}}$.

2) The weight fraction of (ME)PEO block.

3) The ratio between the number of units in PAAm and (ME)PEO blocks.

where $\mathrm{M}_{\mathrm{oPAAm}}, \mathrm{M}_{\mathrm{oPEO}}$ are the molecular weights of PAAm and PEO units, $\mathrm{M}_{\mathrm{nMEPEO}}$ and $\mathrm{M}_{\mathrm{n} P E O}$ are the molecular weights of initial MEPEO and PEO, $\mathrm{A}_{\mathrm{b}}$ and $\mathrm{A}_{c}$ are the integrated intensities of methylene groups of PAAm and (ME)PEO, respectively. Thus, an essentially asymmetric character of synthesized $\mathrm{DBC}$ and $\mathrm{TBC}$ has been established (Table 1).

Some quantity of TBC was partially hydrolyzed to transform a small amount of amide groups into the carboxylic ones, thus enhancing the interaction strength between positively charged molecules of doxorubicin hydrochloride and TBC micelle carrier.

(ME)PEO and PAAm blocks in DBC and $\mathrm{TBC}$ are chemically complementary and form the intramolecular polycomplexes (IntraPCs) due to cooperative hydrogen bonds [23, 24]. This stipulates peculiarities of their synthesis (appearance of the dynamic and structural template effects in the block copolymerization processes) and leads to the formation of special micelle structures in dilute aqueous solutions, which ones contain a complex "core" and stabilizing "corona" composed of surplus (unbound) PAAm segments $[21,22]$. It should be noted that (ME)PEO and PAAm chains are known as non-toxic and biocompatible polymeric components; the first of them can even be biodegraded in living organisms. Therefore, the block copolymers consisting of such blocks could successfully be used in the biomedicine.

The hydrogen bond system in TBC and its thermostability were early characterized by FTIR spectroscopy [23, 24]. It was shown that the cooperative interaction between complementary blocks in TBC macromolecules that is responsible for micelle formation was realized with participation of trans-multimers of PAAm amide groups and oxygen atoms of PEO. In the present work, studies of the hydrogen bond system in $\mathrm{TBC}_{\mathrm{hydr}}$ sample were carried out. FTIR spectra of $\mathrm{TBC}_{\mathrm{hydr}}$ and pure PAAm (for a comparison) at different temperatures are shown in Figure 2. In the spectrum of partially hydrolyzed $\mathrm{TBC}_{\mathrm{hydr}}$ in addition to traditional bands of $v_{\mathrm{C}=\mathrm{O}}$ (amide I), $\delta_{\mathrm{N}-\mathrm{H}}$ (amide II) (Figure 2 a) and $v_{\mathrm{N}-\mathrm{H}}(-$ $\mathrm{CONH}_{2}$ ) (Figure 2 б) vibrations, which were 

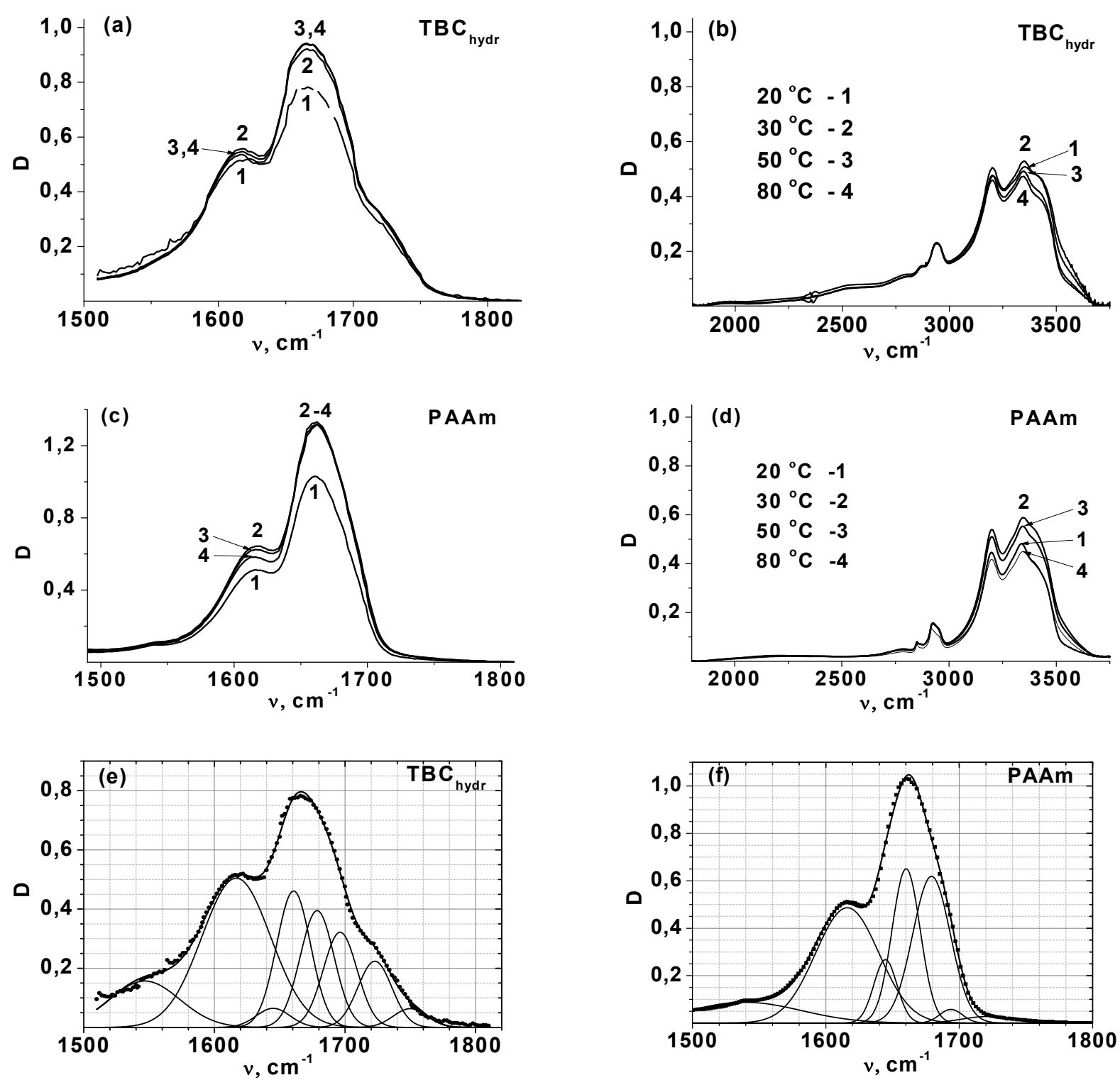

Figure 2. FTIR spectra of thin films $(1 \sim 10 \mu \mathrm{m})$ of $(a, b) \mathrm{TBC}_{\text {hydr }}$ and $(c, d)$ pure PAAm in two most important regions at different temperatures, and computer processing the amide I, amide II and $v \mathrm{C}=\mathrm{O}$ vibration regions in the spectra of $(e) \mathrm{TBC}_{\text {hydr }}$ and $(f)$ pure PAAm at $\mathrm{T}=20^{\circ} \mathrm{C}$

presented in the spectrum of pure PAAm (Figure 2 c, d) and TBC $[23,24]$ and belonged to different types of H-bonds of amide groups, new bands of $v_{\mathrm{C}=\mathrm{O}}(-\mathrm{COOH})$ and $v_{\mathrm{O}-\mathrm{H}}(-\mathrm{COOH})$ vibrations appeared at $\sim 1722 \mathrm{~cm}^{-1}$ and $\sim 2550 \mathrm{~cm}^{-1}$, respectively (Figure $2 \mathbf{a}, \mathbf{b})$. These bands were attributed to the H-bonded carboxyl groups formed in
PAAm blocks of the initial TBC due to a partial hydrolysis of acrylamide units [21].

To compare in detail H-bond systems in $\mathrm{TBC}_{\mathrm{hydr}}$ and PAA thin films, the regions of strongly overlapped bands of $v_{\mathrm{C}}=\mathrm{O}$, amide I, and amide II vibrations (Figure $2 \mathbf{a ,} \mathbf{c}$ ) in the spectra recorded at $20{ }^{\circ} \mathrm{C}$ were processed using a computer program WINSPECTRUM. The 
computer processing was based on our previous studies of the hydrogen bond systems in pure PAAm and block or graft copolymers comprised PAAm chains [23, 24]. The results of band separation are shown in Figure $2(\mathbf{e}, \mathbf{f})$. Thus, the following bands were found in the region of $v_{\mathrm{C}=0}$, amide I and amide II vibrations: a wide intense amide II band (for H-bonded amide groups) at $1616 \mathrm{~cm}^{-1}$, a weak band of $\delta$ O-H vibrations of the adsorbed water molecules at $1645 \mathrm{~cm}^{-1}$, three amide I bands at 1660,1679 and $1695 \mathrm{~cm}^{-1}$, which corresponded to different H-bonded states of PAAm amide groups [23, 24], and finally two $v_{\mathrm{C}=\mathrm{O}}$ bands at 1722 and $1750 \mathrm{~cm}^{-1}$ which reflected the vibrations of $\mathrm{H}$ bonded and free carboxylic groups, consequently [25]. It should be noted that the band at $\sim 1704 \mathrm{~cm}^{-1}$ referred to the amide I vibrations of free amide groups [25], was absent in both the spectra (Figure $2 \mathbf{e}, \mathbf{f}$ ). The band has been taken into account at the computer processing but its integrated intensity was found to be near zero. This means that all amide groups in $\mathrm{TBC}_{\text {hydr }}$ and PAAm films participated in H-bonding.

The data of band separation were considered further together with analysis of the regions of $v_{\mathrm{O}-\mathrm{H}}, v_{\mathrm{N}-\mathrm{H}}$ and $v_{\mathrm{C}-\mathrm{H}}$ vibrations (Figure $2 \mathbf{b , d}$ ) and our previous results [23, 24]. Due to this, the presence of two H-bond structures as in PAAm as in $\mathrm{TBC}_{\text {hydr }}$ films such as cis-transand trans-multimers of amide groups (structures 1, 2) has been confirmed. An additional H-bond structure such as the mixed cyclic dimmer of amide and carboxylic groups (structure 3) was established in $\mathrm{TBC}_{\text {hydr }}$ film. Formation of these

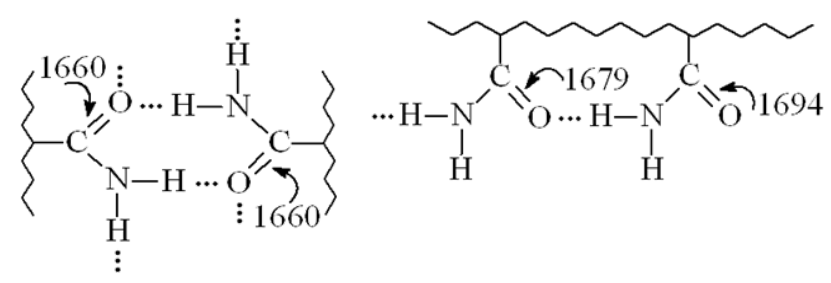
1 2

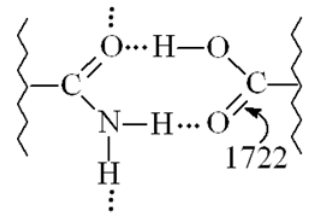

3

cyclic dimmers was proved by the appearance of $v_{\mathrm{C}=\mathrm{O}}$ vibration band of the H-bonded carboxylic groups at $1722 \mathrm{~cm}^{-1}$ (Figure $2 \mathbf{a}, \mathbf{c}, \mathbf{e}$, f) and especially a wide band of the H-bonded hydroxyls of - $\mathrm{COOH}$ groups in a range of 2500$2600 \mathrm{~cm}^{-1}$ (Figure $2 \mathbf{b}, \mathbf{d}$ ), which one is typical for cyclic dimmers with participation of carboxylic groups [26]. The amide I band belonging to the H-bonded amide group in the structure $\mathbf{3}$, should be close to a similar band at $1660 \mathrm{~cm}^{-1}$, referring to the structure I; therefore, it could not be observed separately. In the pointed wavenumber region, the band with low intensity at $1750 \mathrm{~cm}^{-1}$ relating to free carboxylic groups was also identified. It is clear that separate carboxylic groups in the partially hydrolyzed PAAm chains of $\mathrm{TBC}_{\text {hydr }}$ could also be integrated in the structure of trans-multimers of amide groups and interact with oxygen atoms of PEO blocks. 
Table 2. Contributions of the integrated intensities of separate bands into the overall intensity of the amide I and $v_{\mathrm{C}=\mathrm{O}}$ vibrations

\begin{tabular}{|c|c|c|c|c|c|c|}
\hline \multirow{3}{*}{ Copolymer } & \multicolumn{5}{|c|}{$\alpha_{\mathrm{i}}{ }^{1)}$} \\
\cline { 2 - 7 } & \multicolumn{3}{|c|}{$-\mathrm{CONH}_{2}$ groups } & \multicolumn{2}{c|}{-COOH groups } & $\beta^{2)}$ \\
\cline { 2 - 7 } & $1660 \mathrm{~cm}^{-1}$ & $1679 \mathrm{~cm}^{-1}$ & $1695 \mathrm{~cm}^{-1}$ & $1722 \mathrm{~cm}^{-1}$ & $11750 \mathrm{~cm}^{-1}$ & \\
\hline PAAm & 0.41 & 0.53 & 0.03 & 0.03 & 0 & 17.7 \\
\hline $\mathrm{TBC}_{\text {hydr }}$ & 0.31 & 0.27 & 0.22 & 0.15 & 0.04 & 1.2 \\
\hline
\end{tabular}

1) $\alpha_{i}=A_{i} / \Sigma A_{i}$, where $A_{i}$ is the integrated intensity of corresponding band.

2) The effective length of the trans-multimers of amide groups

Unfortunately, existence of the last single bonds can not be confirmed by FTIR spectroscopy data because of small PEO blocks weight fraction in the initial TBC sample (Table 1) that is reflected in a very small intensity of $v_{\text {asCOC }}$ and $v_{\mathrm{SCOC}}$ vibration bands responsible to $\mathrm{H}$ bonding in a region of $1000-1250 \mathrm{~cm}^{-1}$ (they are not shown). The distribution of amide and carboxylic groups between different H-bond structures was characterized (to a first approximation) by the values $\alpha_{\mathrm{i}}$ in Table 2 . The effective length $(\beta)$ of the trans-multimers of amide groups (structure 2) was also defined using known relation: $\beta=A_{1679} / A_{1694}[23,24]$.

According to Table 2, an additional feature of the hydrogen bond system in $\mathrm{TBC}_{\text {hydr }}$ film as compared to PAAm can be noted. Indeed, the $\mathrm{TBC}_{\text {hydr }} \mathrm{H}$-bond system included essentially larger number of the trans-multimers represented by structure 2 , but their length was significantly smaller than in PAAm film. Such conclusion is based on a sharp increase in $\alpha_{i}$ value for the band $1695 \mathrm{~cm}^{-1}$, whose intensity is determined by the amount of trans-multimers and a sharp decrease in $\beta$ number depending on the trans-multimer length.

The H-bond systems in $\mathrm{TBC}_{\text {hydr }}$ and

PAAm demonstrate a high thermostability in the range $20 \div 80^{\circ} \mathrm{C}$. At $\mathrm{T}>30^{\circ} \mathrm{C}$ only small gradual decrease in the intensity of separate vibration bands practically without alterations in their positions was observed (Figure $2 \mathbf{b}$, d), thus indicating a destruction of the negligible amount of the corresponding $\mathrm{H}$-bond structures at the temperature increase. At the same time, a sharp increase in the intensity of all characteristic vibration bands in both the regions was observed at the temperature enhance from 20 to $30{ }^{\circ} \mathrm{C}$ (Figure 2 a-d). It means the growth of the film structure rigidity in the pointed temperature region. Such effect could be connected with an increase in the copolymer segment mobility at $30{ }^{\circ} \mathrm{C}$ that led to their reorientation in space and formation of the largest amount of $\mathrm{H}$-bonds between them.

Thermodynamic parameters and stability of the block copolymer micelles in aqueous solutions were compared by determination of the critical micellization concentration (CMC) 

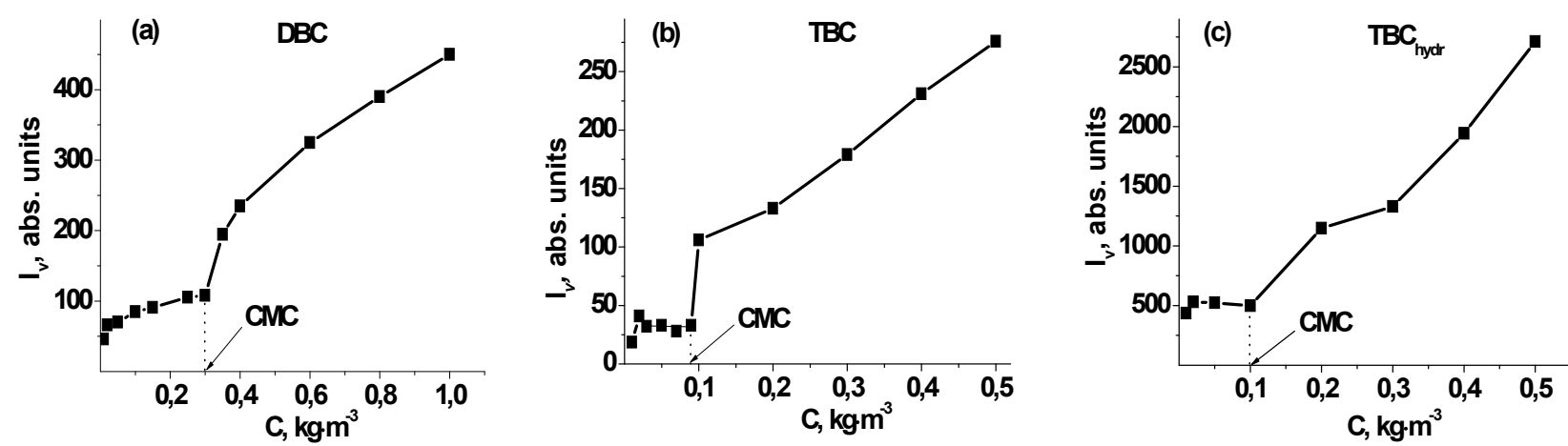

Figure 3. Dependences of the absolute intensity of the vertically-polarized incident light vs concentration in aqueous solutions of (a) DBC, (b) TBC, and (c) $\mathrm{TBC}_{\text {hydr }} . \lambda=520 \mathrm{~nm}, \theta=90^{\circ}$.

Table 3. Thermodynamic parameters of the copolymer micelles

\begin{tabular}{|c|c|c|}
\hline Copolymer & $\mathrm{KKM} \cdot 10^{7}, \mathrm{~mol} \cdot \mathrm{dm}^{-3}$ & $-\Delta \mathrm{G}^{\circ}, \mathrm{KJ} \cdot \mathrm{mol}^{-1}$ \\
\hline DBC & 13.19 & 32.98 \\
\hline TBC & 3.78 & 36.15 \\
\hline TBC $_{\text {hydr }}$ & 3.98 & 36.14 \\
\hline
\end{tabular}

and the Gibbs free micellization energy $\left(-\Delta G^{\circ}\right)$ using static light scattering. The results of CMC finding are shown in Figure 3 and the $-\Delta G^{\circ}$ values were calculated by the (3) relation [27]:

$$
\Delta \mathrm{G}^{\circ} \approx \mathrm{RT} \cdot \ln (\mathrm{CMC})
$$

The obtained micelle parameters are collected in Table 3. As shown, all the copolymer micelles were characterized by very small CMCs and large values of the Gibbs micellization energy, thus indicating a high stability of $\mathrm{DBC}, \mathrm{TBC}$ and $\mathrm{TBC}_{\text {hydr }}$ micelles in aqueous solutions and the great prospects of their use to encapsulate and deliver poorly soluble and/or toxic drugs in the living organism. The essentially less CMC values and higher $-\Delta G^{\circ}$ numbers were found for TBC and $\mathrm{TBC}_{\text {hydr }}$ micelles as compared to DBC ones; therefore, the last micelles were much less stable in water. The values of CMC and $\Delta \mathrm{G}^{\circ}$ for $\mathrm{TBC}_{\text {hydr }}$ micelles turned out to be practically the same as the corresponding parameters for the micelles formed by unmodified TBC sample (Figure 3 b, c; Table 2). Thus, a small fraction of the carboxylic groups in PAAm blocks of TBC macromolecules did not affect the stability of their micelles.

Morphological features and size of DBC and TBC were determined by TEM (Figure 4). The electron images demonstrated not only relatively large and mainly spherical micelles of a polymolecular type with a size of $10 \div 31 \mathrm{~nm}$ for $\mathrm{DBC}$ and $80 \div 240 \mathrm{~nm}$ for TBC, but also the micelles of the monomolecular type (small dark points in the images) with a diameter of $3 \div 6 \mathrm{~nm}$ for 


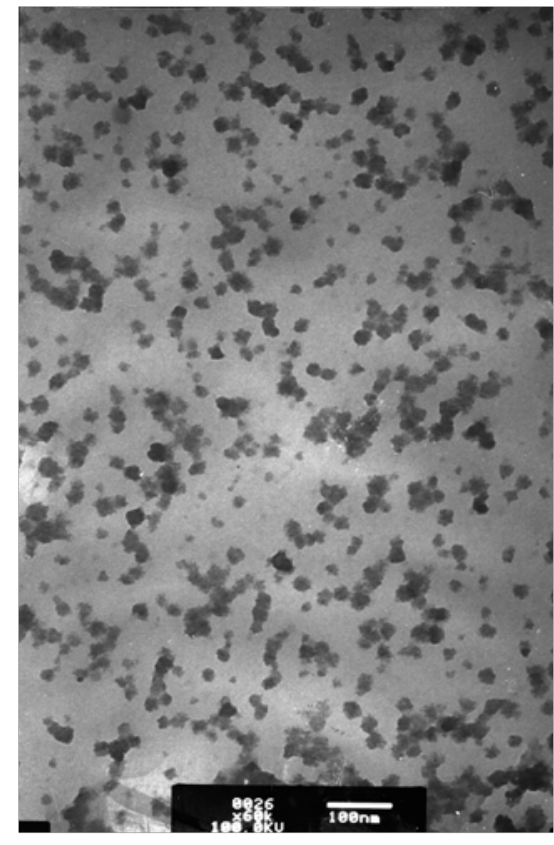

a

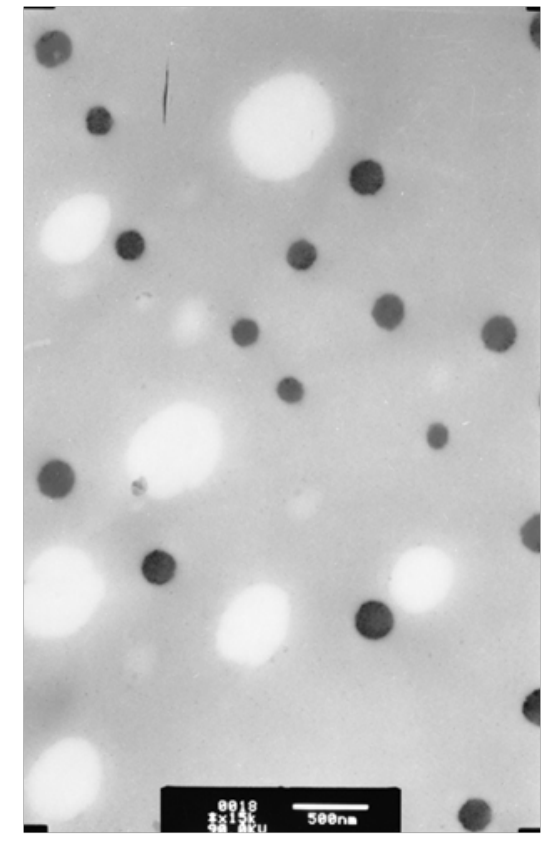

b

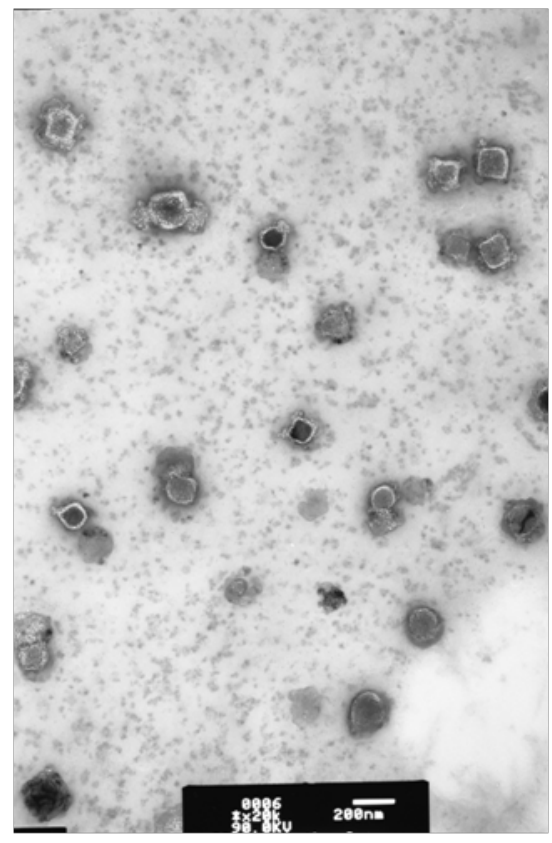

C

Figure 4. TEM images for (a) DBC and (b, c) TBC micelles at the lesser (b) and higher (c) magnification in electron microscope. $\mathrm{C}_{\mathrm{DBC} / \mathrm{TBC}}=0.2 \mathrm{~kg} \cdot \mathrm{m}^{-3}$

DBC and 15 $\div 30 \mathrm{~nm}$ for TBC (Figure $4 \mathbf{a}, \mathbf{c}$ ), which were in fact the individual IntraPCs. Polymolecular micelles of DBC and TBC had a large "corona" due to which they could be attributed to so-called "hairy" micelles [27]. TEM images showed also separate micelle aggregates resulted from combining their "coronas".

In order to encapsulate the anticancer drug Dox, we used aqueous solutions of the block copolymers with $\mathrm{C}=1 \mathrm{~kg} \cdot \mathrm{m}^{-3}>\mathrm{CMC}$, which contained both the mono- and polymolecular micelles. The molecules of Dox hydrochloride contained the developed hydrophobic part, as well as hydroxyl, carbonyl, ether and positively charged amine groups; therefore, one could expect their active interaction with the micelles of $\mathrm{DBC}$,
TBC and especially $\mathrm{TBC}_{\mathrm{hydr}}$. UV-Vis spectroscopy was used to confirm the interaction of Dox with the block copolymer micelles in aqueous solutions.

UV-Vis spectrum of Dox in aqueous solution (Figure 5, spectrum 1) showed 5 intense absorption bands at 233, 253, 289, 480 and $495 \mathrm{~nm}$ due to the presence of "anthraquinone" fragment in its molecule with a system of $\pi$ and $n$ electrons. In the spectrum of DBC micellar solution (Figure 5 a, spectrum 3), only a weak absorption band in the region of 240-300 nm, which could be attributed to the $n \rightarrow \pi^{*}$ electron transition in $-\mathrm{CONH}_{2}$ groups, was present. Thus, changes in the spectral characteristics of Dox in composition with the copolymer micelles could give useful information about its 

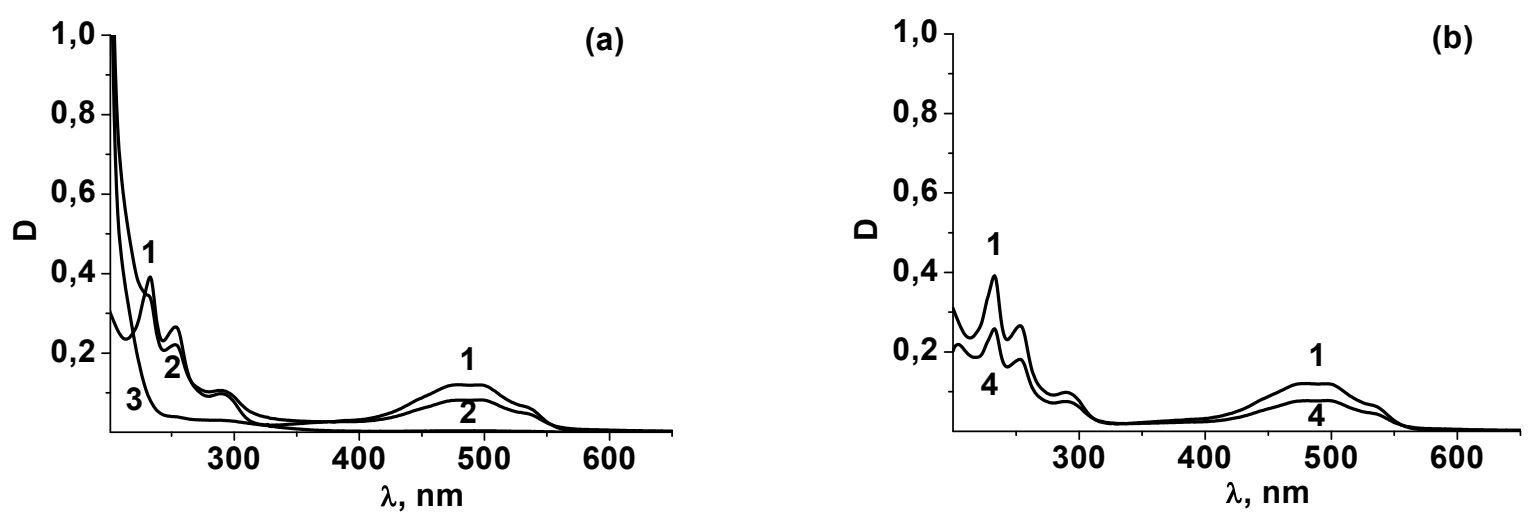

Figure 5. UV spectra of aqueous solutions of (a, b) Dox -1 , (a) Dox/DBC composition -2 and pure DBC -3 as well as (b) the difference in the extinction spectra (Dox/DBC-DBC) $-4 . C_{\text {Dox }}=2,5 \cdot 10^{-5} \mathrm{~mol} \cdot \mathrm{dm}^{-3}$, $\mathrm{C}_{\mathrm{DBC}}=0,12 \mathrm{~kg} \cdot \mathrm{dm}^{-3}, \mathrm{~T}=20^{\circ} \mathrm{C}$.

Table 4. Absorption bands in UV-Vis spectra of pure Dox and its composition with DBC micelles

\begin{tabular}{|c|c|c|c|c|c|c|}
\hline System & Solvent & \multicolumn{5}{|c|}{$\lambda_{\max }, \mathrm{nm}$} \\
\cline { 1 - 5 } Dox & \multirow{2}{*}{$\mathrm{H}_{2} \mathrm{O}$} & 233 & 253 & 289 & 480 & 495 \\
\cline { 4 - 7 } & & 233 & 253 & 290 & 482 & 497 \\
\hline
\end{tabular}

interaction with the nanocarriers. The alterations in the intensity and position of the Dox absorption bands could be traced by comparing UV-Vis spectra of pure Dox and free carriers with the spectrum of the composition. The result of such comparison is shown in Figure $5 \mathbf{b}$ and Table 4. Thus, the bathochromic shift for many adsorption bands of Dox in the composition with DBC micelles (Table. 4) and a decrease in the intensity of these bands (Figure 5 b) have been established. The noted differences in the band positions and intensities reflected the changes in the electron density distribution in Dox molecules under the effect of their interaction with DBC micelles.
Taking into account the higher stability of $\mathrm{TBC}$ and $\mathrm{TBC}_{\mathrm{hydr}}$ micelles in aqueous solutions, as compared to $\mathrm{DBC}$, one exactly two first kinds of micelles were used to examine in vitro anticancer activity of their compositions with Dox. Micellar compositions were prepared at $\mathrm{C}_{\mathrm{TBC}}=0.5-1.0$ $\mathrm{kg} \cdot \mathrm{m}^{-3}$ and the ratio $\varphi=0.01-0.02$ $\mathrm{mol}_{\text {Dox }} \cdot$ base- $\mathrm{mol}_{\mathrm{TBC}}{ }^{-1}$ and then they were diluted as it was necessary. These experiments allowed comparing the cytotoxicity of pure drug, its compositions with $\mathrm{TBC}$ and $\mathrm{TBC}_{\text {hydr }}$ micelles, and free nanocarriers. First of all, we studied a cytotoxicity of pure $\mathrm{TBC}_{\text {hydr }}$ solutions using the human T-leukemia cells (Figure 6). We did not observe any effect at minimum 


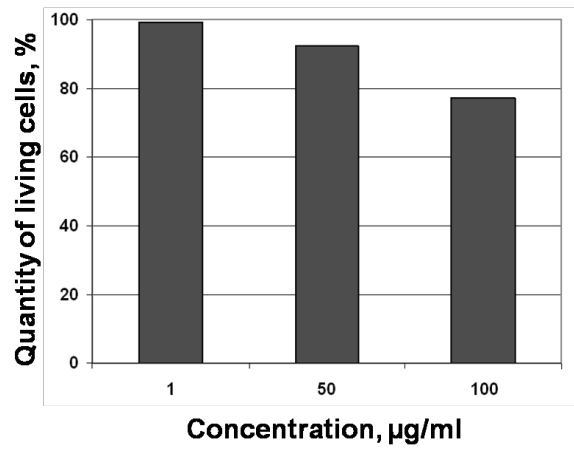

Figure 6. The action of $\mathrm{TBC}_{\mathrm{hydr}}$ on the number of living human T-leukemia cells. The incubation time is $24 \mathrm{~h}$.

$\mathrm{C}_{\mathrm{TBC}}=1 \mu \mathrm{g} \cdot \mathrm{cm}^{-3}$. A noticeable decrease in the number of living human T-leukemia cells appeared at $\mathrm{C}_{\mathrm{TBC}}=50 \mu \mathrm{g} \cdot \mathrm{cm}^{-3}$ but at the maximum $\mathrm{C}_{\mathrm{TBC}}=100 \mu \mathrm{g} \cdot \mathrm{cm}^{-3}$ the reduction in the number of living cells consisted more than $20 \%$.

Different results are presented in Figure 7-9. They show that after incubation for $24 \mathrm{~h}$ a cytotoxic effect of Dox in the composition with $\mathrm{TBC}_{\text {hydr }}$ micelles increased with growth of $\mathrm{C}_{\text {Dox }}$ from 0.1 to $1 \mu \mathrm{g} \cdot \mathrm{cm}^{-3}$ and turned out to be higher as compared to free Dox and Dox composition with the micelles of unmodified TBC (Figure 7 a).

$\mathbf{a}$

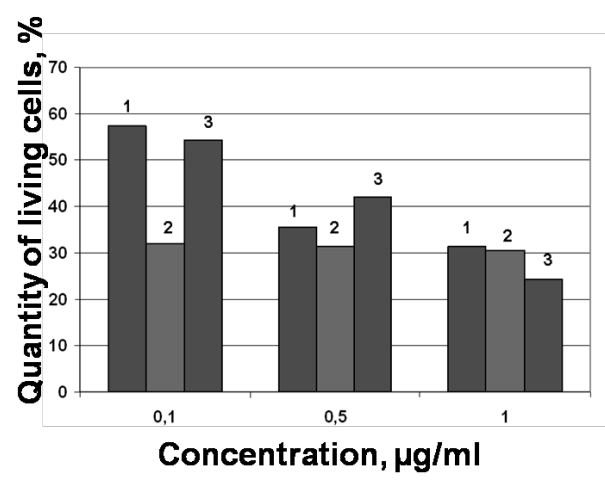

As one can see, the pure Dox displayed lower cytotoxic activity than Dox incorporated into the $\mathrm{TBC}$ and $\mathrm{TBC}_{\mathrm{hydr}}$ micelles at the concentration $1 \mu \mathrm{g} \cdot \mathrm{cm}^{-3}$ only. The result obtained after $48 \mathrm{~h}$ of incubation (Figure 7 b) convinces that Dox/TBC and Dox/TBC effective (in 2.8-4.2 times) than free Dox practically in the all range of concentrations under study.

A similar regularity was observed under the action of free and encapsulated Dox on murine leukemia L1210 cells (Figure 8). In these experiments the most effective was Dox/TBC After $24 \mathrm{~h}$ incubation, the Dox efficiency in micellar media was higher than the efficiency of free drug in 1.70, 1.79 and 1.83 times, respectively, at concentrations of $0.01,0.1,1,3 \mu \mathrm{g} \cdot \mathrm{cm}^{-3}$ (Figure 8 a). After 48 $\mathrm{h}$ of incubation Dox-loaded compositions show the more efficacy (in 1.93, 3.40 i 4.50 times) at concentrations mentioned below

b

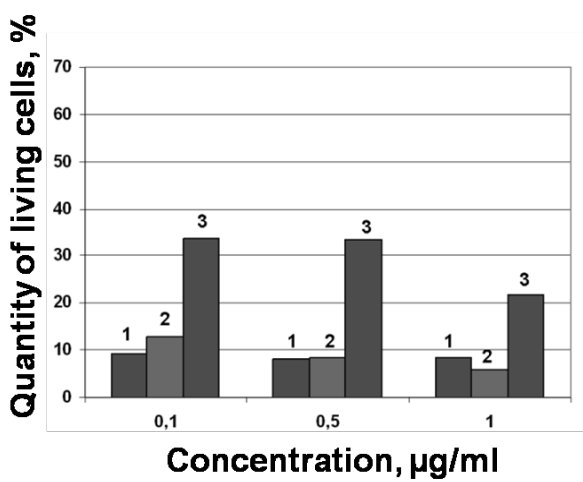

Figure 7. Changes in the number of the living human T-leukemia cells under the effect of pure Dox -1 and its compositions with TBC micelles -2 and $\mathrm{TBC}_{\mathrm{hydr}}$ micelles -3 . The incubation time is 24 (a) and $48 \mathrm{~h}$ (b). 
a

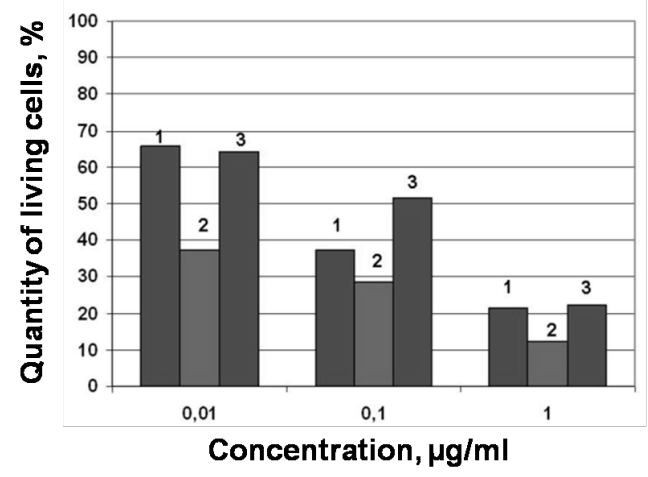

b

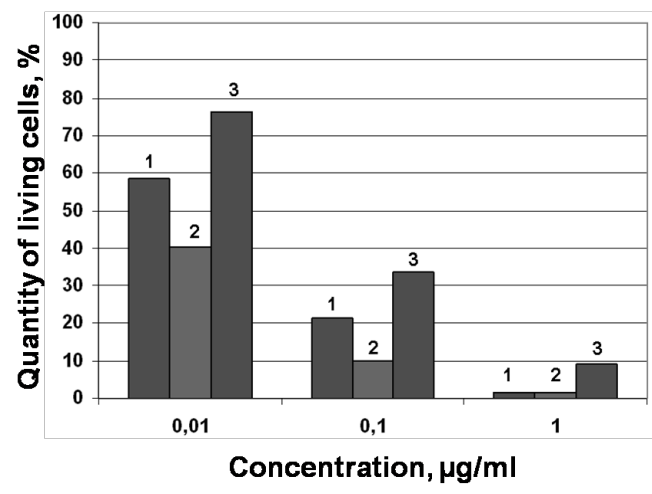

Figure 8. Changes in the number of the living murine L1210 leukemia cells under the effect of pure Dox 1 and its compositions with $\mathrm{TBC}-2$ and $\mathrm{TBC}_{\text {hydr }}$ micelles -3 . The incubation time is 24 (a) and $48 \mathrm{~h}(\mathbf{b})$.

(Figure 8 b). Importantly, it was found that incorporated into the micellar media after $48 \mathrm{~h}$ of incubation both Dox-loaded operates more efficiently than the free form micellar compositions at Dox concentration $1 \mu \mathrm{g} \cdot \mathrm{cm}^{-3}$ caused almost a complete loss of tumor cells viability as opposed to the action of pure Dox.

Studying the dynamics of living cells transformed L929 fibroblasts in the presence of Dox/TBC and Dox/TBC $/ \mathrm{Cydr}_{\text {micellar }}$ compositions, also testified the fact that Dox

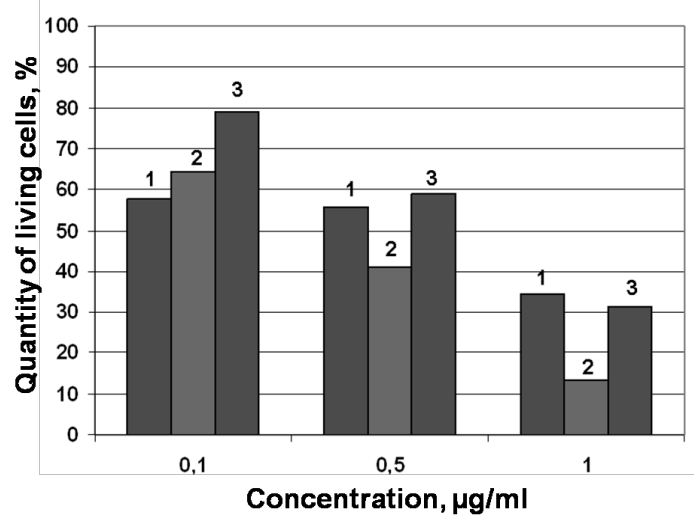

Figure 9. Changes in the number of the living transformed L929 fibroblasts under the action of pure Dox -1 and its compositions with TBC micelles -2 and $\mathrm{TBC}_{\mathrm{hydr}}$ micelles -3 . The incubation time is $24 \mathrm{~h}$. of this drug (Figure 9). Meanwhile, the Dox/TBChydr micellar system shows a tendency to kill tumor cells more actively. The effectiveness of its action exceeded the effect of free Dox in 1.23, 1.44 and 2.37 times at concentrations of anticancer drug $0.1,0.5$ and $1 \mu \mathrm{g} \cdot \mathrm{cm}^{-3}$, respectively.

Dox is the most common anticancer drug in clinical application, and thus it attracts a big attention of researchers creating different macromolecular systems for drug delivery. For example, in the study [28] poly(styrene-alt-maleic acid) (SMA) was used to construct micelles containing Dox by means of the hydrophobic interactions between styrene moieties and Dox. The cytotoxic effect of Dox/SMA composition against human colon cancer SW480 cell line turned out to be lower (about $40 \%$ ), as compared to free Dox after 
$72 \mathrm{~h}$ incubation. Authors attributed this the growth of control cancer cells result to slow uptake into the cells and maintained in the same culture medium strong hydrophobic interactions between without drug. Such experimental conditions Dox and SMA micelles, thus resulting in the corresponded to those described in the slow drug release rate and a delay in the bioavailability of free drug to cells in the culture medium. Other Dox-loaded system based on the poly(ethylene glycol)-blockpolycaprolactone micelles was proposed and examined in the presence of adriamycinresistant K562 tumor cells [29]. The viability of these cells was dramatic, and no significant differences were observed after their incubation with either Dox or Doxloaded micelles for 3 days in the range of Dox concentrations from 3 to $12 \mu \mathrm{g} \cdot \mathrm{cm}^{-3}$. In the adriamycin-resistant K562 tumor cells, notably enhanced cytotoxicity was induced by the drug-loaded micelles, as compared to pure Dox at $\mathrm{C}_{\text {Dox }}=6 \div 12 \mu \mathrm{g} \cdot \mathrm{cm}^{-3}$. At the same time, the physical mixture of polymer and Dox showed the same cytotoxicity as the Dox solution.

Alternative Dox/polymer conjugates based on poly (amidoamines), water-soluble copolymers based on N-(2hydroxypropyl)methacrylamide, copolymers compraising cholic acid and polyethyleneimine have been presented for Dox delivery $[28,30,31]$. As a rule, the cytotoxicity was assessed relatively to free Dox after 24, 48, and $72 \mathrm{~h}$ incubation and signified as viability expressed as percent of present paper; therefore, it is possible to compare the results. In most cases the toxicity of the Dox/polymer conjugates was significantly less than of pure Dox that was explained by a slower endocytotic mechanism of uptake, as compared to rapid diffusion of free Dox. At the same time, authors of the study [31] observed an enhanced cytotoxicity of the Dox-loaded micelle conjugates in the presence of human colorectal adenocarcinoma DLD-1 cells and Chinese hamster lung fibroblast V79 cells. Namely, 60-89 \% inhibition of cancer cells against $65 \%$ for pure drug at a top Dox concentration of $5 \cdot 10^{-2} \mathrm{mg} \cdot \mathrm{cm}^{-3}$. It should be noted that synthesis of Dox/polymer conjugates is a multi-step and rather complicated process. On the contrary, DBCs and TBCs presented in this paper are prepared in aqueous solutions without any toxic organic solvents. On the other hand, Dox/TBC and Dox/TBChydr micelle compositions allow achieving a high efficacy at low Dox concentrations $(0,1 \div 3$ $\mu \mathrm{g} \cdot \mathrm{cm}^{-3}$ ) that opens the prospects for an essential decrease in drug dose at the chemotherapy.

\section{Conclusions}

The polymeric micelles of a special type 
were obtained and evaluated as potential carriers for anticancer agent doxorubicin. The micelles were generated by asymmetric triblock (TBC) and diblock (DBC) copolymers containing biocompatible chemically complementary polyacrylamide and poly(ethylene oxide) (PAAm- $b$-PEO- $b$-PAAm) or its monomethyl ether (MEPEO- $b$-PAAm). Separate fraction of TBC was also transformed into partially hydrolyzed $\mathrm{TBC}_{\text {hydr }}$ to strengthen the interaction between positively charged molecules of doxorubicin hydrochloride and TBC micelle carrier due to appearance of certain quantity of carboxylic groups in PAAm links.

Obtained micelles are stable in aqueous solutions although DBC ones displayed less stability as compared to TBC. The creation of a small fraction of carboxylic groups in PAAm blocks of TBC macromolecules did not affect the stability of their micelles, which displayed in a very similar $\mathrm{CMC}$ and $-\Delta \mathrm{G}^{\circ}$ values of $\mathrm{TBC}$ and TBC $C_{\text {hydr }}$.

Morphology studies revealed the presence of polymolecular and monomolecular micelles of a spherical shape. Polymolecular micelles of DBC and TBC had a large "corona" composed of surplus PAAm segments due to which they could be attributed to the "hairy-type" micelles, while monomolecular ones were in fact the individual IntraPCs.
Biological investigations in vitro

demonstrated essential anticancer activity of Dox/TBC and Dox/TBC hydr $_{\text {compositions, as }}$ compared to pure Dox in murine and human leukemia cells. The ability to decrease a therapeutic dose of doxorubicin in 2-5 times and thus to reduce significantly its toxic effect on normal organs and tissues have been considered.

\section{Acknowledgements}

The authors are grateful for financial support provided by National Taras Shevchenko University of Kyiv. Additionally, this work was also partially supported by the Institute of Cell Biology and Institute of Botany (National Academy of Science of Ukraine).

\section{References}

[1] Torchilin V. Nanoparticulates as drug carriers. Imperial College Press, London; 2006, pp.58-62.

[2] Mahmud A, Xiong XB, Aliabadi HM, Lavasanifar A L. Polymeric micelles for drug targeting. J. Drug Target 2007;15:553-584

[3] Tong R, Cheng J. Anticancer Polymeric Nanomedicines. Polym. Rev 2007;47:345-381

[4] Attia AB, Ong ZY, Hedrick L, Lee PP, Ee PL, Hammond PT,YangYY. Mixed micelles selfassembled from block copolymers for drug delivery. Curr. Opin. Coll. Int. Sci 2011;16:182-194

[5] Osada K, Christie RJ, Kataoka KJ. Polymeric micelles from poly(ethylene glycol) poly(amino acid) block copolymer for drug and gene delivery. J. Royal Soc. Inerface 2009; 6:S325-S339

[6] Dane KY, Nembrini CA, Tomei AA, Eby JK, O’Neil CP, Velluto D, Swartz MA, Inverardi L, Hubbell JA. Nano-sized drug-loaded micelles deliver 
payload to lymph node immune cells and prolong allograft survival. J. Contr. Release 2011;156: 154-160

[7] Ohya Y, Takahashi A, Nagahama K. Biodegradable polymeric assemblies for biomedical materials. Adv. Polym. Sci 2012;247:65-114

[8] Zhang Q, Ko NR, Oh JK. Recent advances in stimuli-responsive degradable block copolymer micelles: synthesis and controlled drug delivery application. Chem. Commun 2012;48:7542-7552

[9] Sutton D, Nasongkla N, Blanco E, Gao J. Functionalized micellar systems for cancer targeted drug delivery. Pharm. Res 2007; 24:1029-1046

[10] Wang YC, Liu XQ, Sun TM, Xiong $\mathrm{MH}$, Wang J. Functionalized micelles from block copolymer of polyphosphoester and poly(E-caprolactone) for receptor mediated drug delivery. J. Control. Release 2008; $128: 32-40$

[11] Noh T, Kook YH., Park C, Youn H, Kim H, Oh ET, Choi EK, Park HJ, Kim C. Block copolymer micelles conjugated with anti-EGFR antibody for targeted delivery of anticancer drug. J. Polym. Sci.: Part A: Polym. Chem 2008;46:7321-7331

[12] Shi M, Lu J, Shoichet MS. Organic nanoscale drug carriers coupled with ligands for targeted drug delivery in cancer. J. Mater. Chem 2009;19: 54855498

[13] Freichels H, Jerome R, Jerome C. Sugar-labeled and PEGylated (bio)degradable polymers intended for targeted drug delivery systems. Carbohydrate Polym 2011 ;86 : 1093-1106

[14] Chatterjee K, Zhang J, Honbo N, Karlinerb J S. Doxorubicin cardiomyopathy. Cardiology 2010;115(2):155-162

[15] Sakthibalan M, Sawadkar MS, Asmathulla S, Ivan EA, Muthu G. Study of cardioprotective effect of $\mathrm{N}$-acetilcysteine, Vitamine $\mathrm{C}$ and Enalapril given incombination to prevent doxorubicin induced cardio toxicity in Wistar rats. Pharm. Biomed. Sci 2013;36:1902-1908
[16] Glen S, Marcus L. inventor; Wisconsin Alumni Research Foundation, assignee. Micelle composition of polymer and passenger drug. United States patent 8173 167. 2012 May 8.

[17] Xu Y, Meng F, Cheng R, Zhong Z. Reduction-sensitive reversibly crosslinked degradable micelles for triggered release of doxorubicin. Macromol. Biosci 2009;9:1-7

[18] Heller G, Ng SY, inventors; Advanced Polymer Systems, Inc., assignee. PEG-POE, PEG-POE-PEG and POEPEG-POE block copolymers. United States Patent 5939453.1999 Aug 17.

[19] Kim JO, Kabanov AV, Bronich TK. Polymer micelles with cross-linked polyanion core for delivery of a cationic drug doxorubicin. J. Control. Release 2009;138:197-204

[20] Chan Y, Wong T, Byrne F, Kavallaris M, Bulmus V. Acid-labile core cross- linkedm icelles for $\mathrm{pH}-$ triggered release of antitumor drugs. Biomacromol 2008;9:1826-1836

[21] Fedorchuk SV, Zheltonozhskaya TB, Pemyakova NM, Gomza YP, Nessin SD, Klepko VV. Structural peculiarities of triblock copolymers containing poly(ethylene oxide) and polyacrylamide. Mol. Cryst. Liq. Cryst 2008;497: 268-391

[22] Zheltonozhskaya T, Nedashkovskaya V, Khutoryanskiy V, Gomza Yu, Fedorchuk S, Klepko V, Partsevskaya S. Micelles of PAAm- $b$-PEO- $b$-PAAm triblock copolymers and their binding with prednisolon. Mol. Cryst. Liq. Cryst 2011;536:380-391

[23] Zheltonozhskaya TB, Permyakova NN, Momot LN. Intramolecular polycomplexes in block and graft copolymers. In: Khutoryanskiy V, Staikos G, editors. Hydrogen-Bonded Interpolymer Complexes. Formation, Structure and Application. New Jersey - London Singapore :World Scientific; 2009. P. 85-153.

[24] Permyakova NM, Zheltonozhskaya TB, Fedorchuk SV, Zagdanskaya NE, Syromyatnikov VG. Temperature effect on hydrogen bonds in triblock 
copolymers of poly(ethylene oxide) and polyacrylamide.

Mol. Cryst. Liq. Cryst 2007;468:53-61

[25] Pretsch E, Bullman F, Affolter C, Structure determination of organic compounds. Tables of spectral data. Moscow: Mir; 2006, pp.157-242.

[26] Bellamy LJ, The Infra-red Spectra of Complex Molecules. London: Chapman and Hall; 1975, pp.86-115.

[27] Riess G. Micellization of block copolymers. Progress in Polymer Science; 2003, pp.1107- 1170.

[28] Lavignac N, Nicholls JL, Ferrutti P, Duncan R. Poly(amidoamine) conjugates containing doxorubicin bound via an acid-sensitive linker. Macromol. Biosci 2009;9:480-487

[29] Diao Y, Li H, Fu Y, Han M, Hu Y, Jiang H, Tsutsumi Y, Wei Q, Chen D, Gao G. Doxorubicin-loaded PEG-PCL copolymer micelles enhance cytotoxicity and intracellular accumulation of doxorubicin in adriamycinresistant tumor cells. Int. J. Nanomedicine 2011;6: 19551962

[30] Zarabi B, Nan A, Zhuo J, Guallapalli R, Ghandehari H. HPMA Copolymer- doxorubicingadolinium conjugates: synthesis, characterization and in vitro evaluation. Macromol. Biosci 2008;8: 741-748

[31] Amjad M, Amin M, Katas H, Butt A. Doxorubicin-loaded cholic acid-polyethyleneimine micelles for targeted delivery of antitumor drugs: synthesis, characterization and evaluation of their in vitro cytotoxicity. Nanoscale Res.Lett 2012;7:687-700 